\title{
Arzneimittelkonsum von Frauen - Ergebnisse der Nationalen Surveys
}

\author{
Von H. Knopf und H.-U. Melchert
}

\section{Zusammenfassung}

Die Daten der nationalen Untersuchungs-Surveys (19841991) belegen in allen analysierten Alters- und Arzneimittelklassen (AT-Code) verglichen mit den Männern ein mehr oder weniger deutliches Überwiegen des Arzneimittelverbrauchs bei den Frauen. Neben der Anwendungshäufigkeit von Psychopharmaka sind diese Unterschiede besonders groß bei Präparategruppen, die nicht der Rezeptpflicht unterliegen wie z.B. Laxanzien, Vitamine und Schmerzmittel. Bereits vorliegende Publikationen zur Bestimmung ausgewählter Arzneimittelwirkspiegel im Serum der Studienteilnehmer und klinisch-chemischer/hämatologischer Meßwerte geben Auskunft über mögliche Korrelationen zwischen Arzneimittelexposition und physiologischen Parametern. Im Hinblick auf mögliche mutagene und teratogene Wirkungen ist die Arzneimitteleinnahme in der Schwangerschaft und Stillzeit von besonderem Interesse.

\section{Summary}

Drug Usage for Females - Results of the National Surveys

The data of the German National Health-Examination-Surveys (1984-1991) show in all analyzed age- and drug-groups (AT-Code) a more or less clear preponderance of drug usage for females. Besides the frequency of usage for psychotropic drugs the differences in comparison to males are especially pronounced in drug groups which are marketed as OTCdrugs in Germany e.g. laxatives, vitamines and analgesics. Already published studies concerning the determination of selected pharmacologically active substances in serum samples of study participants and clinical chemistry/hematological data show possible correlations between drug exposure and vital physiological parameters. With regard to potential mutagenic or teratogenic effects drug usage is of special concern during pregnancy and breast-feeding.

\section{Einleitung}

Zuverlässige Daten zum tatsächlichen Arzneimittelgebrauch einzelner Bevölkerungsgruppen sind selbst in Ländern mit gut ausgebauten Gesundheitsversorgungssystemen eher spärlich vorhanden. Die Datensammlungen der Krankenversicherer beruhen fast ausnahmslos auf Verschreibungsdaten [1], und die ebenfalls weitgehend verschreibungsorientierten Datensammlungen der pharmazeutischen Hersteller werden bisher überwiegend als Instrumente der Marktforschung und -beeinflussung [2] eingesetzt. Im Zusammenhang mit allgemeinen Fragen der Arzneimittelsicherheit und der Bewertung von eventuell auftretenden oder beobachteten unerwünschten Arzneimittelwirkungen ist die sichere Kenntnis von ArzneimittelExpositionsdaten aber eine unverzichtbare Größe, um sowohl das tatsächliche Ausmaß möglicher Gefährdungen als auch das Ausmaß erwünschter Arzneimittelwirkungen zuverlässig $\mathrm{zu}$ beschreiben. Pharmakoepidemiologische Studien mit repräsentativen Bevölkerungsgruppen sind geeignete Instrumente, um diese bestehenden Wissenslücken zu schließen. Dadurch können sowohl Beiträge zur Erhöhung der Arzneimittelsicherheit geleistet als auch $\mathrm{Da}$ ten zur Pharmakoökonomie zur Verfügung gestellt werden.
Im Rahmen der Nationalen Untersuchungs-Surveys der DHP wurden daher seit 1984 pharmakoepidemiologische Erhebungen durchgeführt, um erstmalig für die Bundesrepublik Deutschland und die neuen Bundesländer Basisdaten des tatsächlichen Arzneimittelgebrauchs für repräsentative Bevölkerungsgruppen zu erhalten. Publikationen von Teildaten wurden in den vergangenen Jahren regelmäßig in den $\mathrm{Be}-$ richten des Bundesgesundheitsamtes [3-5] und einer seiner Nachfolgeeinrichtungen [6-9] sowie als Übersichtsbericht für den gesamten ersten SurveyDurchgang [10] publiziert.

Wie aus verschiedenen Verordnungsund Verkaufsstatistiken bekannt ist, zeigen die Abgabezahlen von Arzneimitteln an Frauen in einer Reihe von Therapiegruppen deutliche Differenzen gegenüber den Abgabezahlen von Arzneimitteln an Männer [1]. Dies gilt für die Mehrzahl aller Therapiegruppen und für die Altersgruppen der bis 69jährigen. Trotz dieser Kenntnis sind spezielle Un- tersuchungen zum tatsächlichen Arzneimittelverbrauchsprofil von Frauen eher selten und beschränken sich häufig auf ausgewählte Therapiegruppen [11, $12]$.

\section{Allgemeine Daten zum Arz- neimittelverbrauch}

In den Datensammlungen der oben erwähnten Untersuchungs-Surveys sind alle Arzneimittel, die von den Studienteilnehmern (Tab. 1) in den letzten sieben Tagen vor der Befragung/Untersuchung angewandt wurden, erfaßt worden. Dies gilt insbesondere auch für freiverkäufliche Präparate. Durch die Verbindung der Arzneimittelerhebung mit den Basisdaten der DHP ergeben sich umfangreiche Möglichkeiten der Auswertung des bestehenden Datensatzes. Insbesondere die soziodemographischen Informationen lassen arzneimittelgruppenorientierte Aussagen zu. Diese sind sowohl für die Gesundheitsberichterstattung als auch für Fragen der

Tabelle 1: Studienteilnehmer der Nationalen Untersuchungs-Surveys (NUS)

\begin{tabular}{llll}
\hline & Gesamt & Männer & Frauen \\
\hline Survey T0 1984-1986 & 4790 & 2417 & 2373 \\
Survey T1 1987-1989 & 5335 & 2649 & 2686 \\
Survey T2 1990-1991 & 5311 & 2688 & 2623 \\
\hline
\end{tabular}


Tabelle 2: Weibliche Arzneimittelanwender nach AT-Klassen und Altersgruppen

\begin{tabular}{|c|c|c|c|}
\hline \multirow{2}{*}{$\begin{array}{l}\text { NUS TO-T2 Arzneimittelanwender } \\
\text { nach AT-Klassen und } \\
\text { Altersgruppen }\end{array}$} & \multirow[t]{2}{*}{ Gesamt } & \multicolumn{2}{|c|}{ Altersgruppen } \\
\hline & & $\begin{array}{l}25-45 \\
\text { Jahre }\end{array}$ & $\begin{array}{l}46-69 \\
\text { Jahre }\end{array}$ \\
\hline Stichprobenumfang & 7719 & 3844 & 3875 \\
\hline $\begin{array}{l}\text { Arzneimittelanwender insgesamt } \\
\text { Arzneimittelanwender in der AT-Klasse }\end{array}$ & $71,8 \%$ & $65,2 \%$ & $78,3 \%$ \\
\hline A00 Alimentäres System und Stoffwechsel & $23,9 \%$ & $16,1 \%$ & $31,8 \%$ \\
\hline BOO Blut und blutbildende Organe & $6,7 \%$ & $2,5 \%$ & $10,8 \%$ \\
\hline COO Kardiovaskuläres System & $26,1 \%$ & $10,4 \%$ & $41,5 \%$ \\
\hline D00 Dermatologika & $5,3 \%$ & $6,3 \%$ & $4,4 \%$ \\
\hline GOO Urogenitalsystem und Sexualhormone & $20,1 \%$ & $27,3 \%$ & $13,1 \%$ \\
\hline Hoo Hormonales System exkl. Sexualhormone & $9,7 \%$ & $8,8 \%$ & $10,6 \%$ \\
\hline Joo Antiinfektiva, systemisch & $1,4 \%$ & $1,6 \%$ & $1,2 \%$ \\
\hline Loo Zytostatika & $0,3 \%$ & $0,1 \%$ & $0,6 \%$ \\
\hline M0O Muskel- und Skelettsystem & $7,7 \%$ & $3,5 \%$ & $11,7 \%$ \\
\hline Noo Zentrales Nervensystam & $19,4 \%$ & $15,7 \%$ & $23,2 \%$ \\
\hline POO Parasitologie & $0,1 \%$ & $0,1 \%$ & $0,1 \%$ \\
\hline R00 Respirationssystem & $7,8 \%$ & $7,8 \%$ & $7,8 \%$ \\
\hline SOO Sinnesorgane & $1,7 \%$ & $0,8 \%$ & $2,5 \%$ \\
\hline Voo Varia & $3,7 \%$ & $2,8 \%$ & $4,6 \%$ \\
\hline Z00 Homöopathika, nicht anders zuordenbar & $1,5 \%$ & $1,3 \%$ & $1,8 \%$ \\
\hline
\end{tabular}

Arzneimittelsicherheit und der Pharmakoökonomie von großem Interesse.

Wie die Tabellen 2 und 3 zeigen, weist der Arzneimittelverbrauch von Frauen, die in ambulanter ärztlicher Behandlung sind oder durch Selbstmedikation den verschiedenen Arzneimitteln gegenüber exponiert sind, einige Besonderheiten gegenüber dem Arzneimittelverbrauch von Männern auf. Diese Unterschiede bilden im wesentlichen die unterschiedliche Morbiditätssituation der beiden Geschlechter ab. Daß der Arzneimittelverbrauch der Frauen erheblich höher ist als der der Männer, gilt für nahezu alle Therapiegruppen. Extrem deutlich wird die unterschiedliche Arzneimittelexposition in den AT-Klassen NOO, HOO und $\mathrm{AOO}$. Auch die Altersabhängigkeit der Arzneimittelanwendung wird aus den Tabellen 2 und 3 deutlich erkennbar. Der Unterschied in der AT-Klasse AOO ist im wesentlichen durch den erheblich höheren Verbrauch an Vitaminpräparaten und der Unterschied in der AT-Klasse HOO durch die stärkere Anwendung von Schilddrüsenhormonen durch Frauen begründet.

\section{Ausgewählte Beispiele für einzelne Therapieklassen bzw. Wirkstoffe}

Wie bereits früher mitgeteilt, gibt es markante Geschlechtsdifferenzen im

Gebrauch der verschiedensten Psychopharmaka [8]. Dies ist auch aus den Serumspiegeln der Benzodiazepine Diazepam, Oxazepam und Nitrazepam zu belegen. Das bei weitem am häufigsten verwendete Benzodiazepin war in allen

NUS T0-T2 Arzneimittelanwender nach AT-Klassen und

Altersgruppen

Stichprobenumfang

Arzneimittelanwender insgesamt

Arzneimittelanwender in der AT-Klasse

A00 Alimentäres System und Stoffwechsel

B00 Blut und blutbildende Organe

COO Kardiovaskuläres System

Doo Dermatologika

G00 Urogenitalsystem und Sexualhormone

J00 Antiinfektiva, systemisch

Loo Zytostatika

MOO Muskel- und Skelettsystem

NoO Zentrales Nervensystem

POO Parasitologie

R00 Respirationssystem

SOO Sinnesorgane

Voo Varia drei Survey-Durchgängen der Wirkstoff Bromazepam.

Zur Häufigkeit der Anwendung von Kontrazeptiva, zur Aufschlüsselung nach den einzelnen Kontrazeptiv-Gruppen, zu soziodemographischen Daten ihrer Anwendung und den erkannten physiologischen Auswirkungen eines Einsatzes dieser Präparate liegen ebenfalls ausführliche Untersuchungen vor [9]

Daß die Anwendung von Laxanzien besonders starke Unterschiede zwischen Frauen und Männern aufweist, zeigt die Abbildung 1. Im ersten Survey-Durchgang (1984-1986) verbrauchten Frauen über fünfmal häufiger Laxanzien als Männer. Diese Situation hat sich unter anderem nach verschiedenen Maßnahmen zur Aufklärung in der Öffentlichkeit deutlich verändert. So war der Laxanzienverbrauch der Frauen im dritten Survey nur noch ca. dreimal so groß wie der der Männer. Weitere Informationen zu diesem Themenkomplex, zu den unterschiedlichen Wirkstoffen der Laxanzien und zu den physiologischen Auswirkungen ihrer regelmäßigen Anwendungen sind bereits publiziert worden [13].

Wie bereits oben erwähnt, liegen auch bei der Anwendung von Vitaminpräparaten drastische Differenzen zwischen Frauen und Männern vor. Die Abbil-

Tabelle 3: Männliche Arzneimittelanwender nach AT-Klassen und Altersgruppen

Gesamt Altersgruppen

HoO Hormonales System exkl. Sexualhormone

$25-45$

46-69

Jahre Jahre

7762

3913

3749

$47,9 \%$

$34,9 \%$

$61,5 \%$

\begin{tabular}{rrr}
$15,3 \%$ & $10,2 \%$ & $20,6 \%$ \\
$5,7 \%$ & $1,5 \%$ & $10,0 \%$ \\
$18,1 \%$ & $5,6 \%$ & $31,2 \%$ \\
$3,7 \%$ & $3,7 \%$ & $3,7 \%$ \\
$2,6 \%$ & $0,6 \%$ & $4,6 \%$ \\
$2,4 \%$ & $1,8 \%$ & $3,1 \%$ \\
$1,2 \%$ & $1,3 \%$ & $1,1 \%$ \\
$0,2 \%$ & $0,1 \%$ & $0,2 \%$ \\
$8,5 \%$ & $4,5 \%$ & $12,7 \%$ \\
$11,0 \%$ & $8,9 \%$ & $13,2 \%$ \\
$0,1 \%$ & $0,2 \%$ & $0,1 \%$ \\
$7,9 \%$ & $6,5 \%$ & $9,3 \%$ \\
$1,2 \%$ & $0,7 \%$ & $1,7 \%$ \\
$1,9 \%$ & $1,7 \%$ & $2,2 \%$ \\
$1,1 \%$ & $0,7 \%$ & $1,5 \%$ \\
\hline
\end{tabular}


dung 2 zeigt dies für alle genannten Präparate deutlich. Für die Anwender von Vitamin $E$ ( $\alpha$-Tocopherol)-Präparaten wurden Serumkonzentrationen dieses Vitamins gemessen. Dabei konnte beobachtet werden, daß unter der regelmäßigen Einnahme von $\alpha$-Tocopherol die Serumgehalte von $\beta$ - und $\gamma$-Tocopherol drastisch vermindert werden. Der beobachtete Effekt ist dosisabhängig und betrifft, bedingt durch die höhere Anwendungsfrequenz, insbesondere Frauen [14].

Wie aus der Tabelle 2 zu erkennen ist, weist die Arzneimittelklasse $\mathrm{NOO}$ für die gesamte Gruppe der Frauen mit 19,4 \% eine sehr hohe Anwendungshäufigkeit auf. In dieser Klasse befinden sich auch die angewandten Analgetika. Diese enthalten nach wie vor relativ häufig als Kombinationspräparate Koffein. Die Abbildungen 3 und 4 zeigen, daß die Häufigkeit der Anwendung koffeinhaltiger Schmerzmittel im Laufe der drei Surveys deutlich abgenommen hat. Die Verwendung salizylsäurehaltiger Arzneimittel zeigt im Verlauf der drei Surveys eine deutliche Zunahme unter den Männern. Im letzten Survey haben sich die Gebrauchszahlen weitgehend angenähert. Dies ist insbesondere auf die gestiegene Anwendung salizylsäurehaltiger Präparate als Thrombozytenaggregationshemmer zurückzuführen. Die erfragten Anwendungszahlen dieser

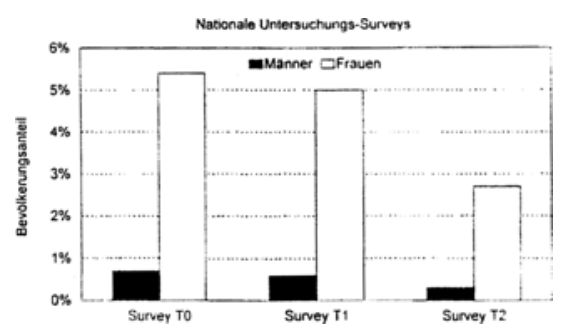

Abbildung 1: Laxanzienverbrauch der 25bis 69jährigen Männer und Frauen.

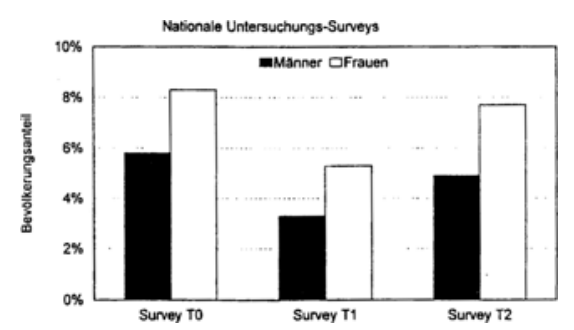

Abbildung 2: Anwendung von Vitaminpräparaten bei 25- bis 69jährigen Männern und Frauen.
Präparate finden ihren Niederschlag in den Serumkonzentrationen von Koffein und Salizylsäure. Die Ergebnisse dieser Untersuchungen wurden jüngst bei der 14. International Conference on Pharmacoepidemiology in Berlin vorgestellt $[15,16]$.

Da für alle Studienteilnehmer nicht nur die Ergebnisse der ärztlichen Untersuchung, sondern auch umfangreiche klinisch-chemische und hämatologische Daten vorliegen, besteht die Möglichkeit, eventuelle Auswirkungen der regelmäßigen oder auch nur gelegentlichen Arzneimitteleinnahme auf wesentliche physiologische Parameter zu untersuchen. Diese Möglichkeit wird noch dadurch erweitert, daß für eine Reihe ausgewählter Arzneimittelwirkstoffe deren Serumkonzentrationen durch chromatographische oder enzymimmunologische Meßverfahren bestimmt wurden. Dies gilt außer für die oben bereits genannten auch für folgende Stoffklassen/Stoffe: Digitalisglykoside (Digoxin/Digitoxin), Theophyllin, Thyroxin, weitere Vitamine und weitere Analgetika und nichtsteroidale Antirheumatika.

\section{Abschließende Betrachtungen}

Da die Anwendung von Arzneimitteln bei Frauen wesentlich häufiger zu verzeichnen ist als bei Männern und da die Einnahmetreue (Compliance) in der Regel deutlich stärker ausgeprägt ist als bei Männern, ist auch davon auszugehen, daß Frauen in stärkerem Maße von möglichen unerwünschten Arzneimittelwirkungen betroffen sind als Männer. Dies wird auch aus den Daten der Surveys deutlich. Anzumerken ist, daß bei der Klassifizierung unerwünschter Arzneimittelwirkungen in einigen Organsystemklassen unerwünschte Arzneimittelwirkungen von Männern häufiger genannt werden als von Frauen [10].

Unter der Berücksichtigung der Tatsache, daß mögliche mutagene bzw. teratogene Wirkungen insbesondere für Frauen im gebärfähigen Alter von erheblicher Bedeutung sind, sollte die Versorgung mit notwendigen Arzneimitteln einer besonders sorgfältigen und sachverständigen Kontrolle unterliegen. Den besonderen physiologischen Umständen wird zur Zeit im wesentlichen dadurch Rechnung getragen, $d a ß$ in den einschlägigen Informationswerken auch auf solche Fragen eingegangen wird. Zuverlässige Informationen über das Auftreten pharmakologisch wirksamer Stoffe bzw, ihrer Metaboliten in der

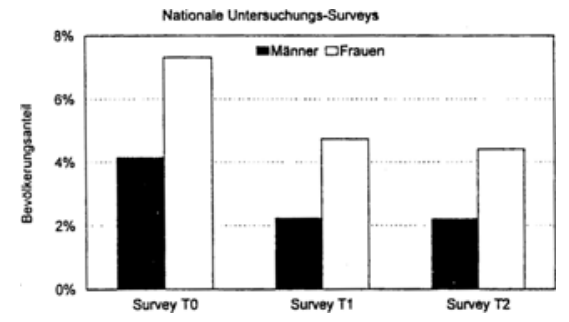

Abbildung 3: Anwendung von koffeinhaltigen Arzneimitteln bei 25- bis 69jährigen Männern und Frauen.

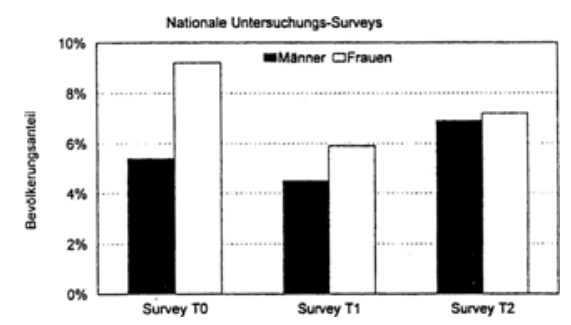

Abbildung 4: Anwendung von salizylsäurehaltigen Arzneimitteln bei 25- bis 69 jährigen Männern und Frauen.

Muttermilch sind unverzichtbar, um mögliche schädliche Auswirkungen der Arzneimittelanwendung auf Mutter und Kind zu verhindern. Hier sei nur eine der neueren Übersichtspublikationen dazu erwähnt [17].

Trotz der höheren Prävalenz von Krankheiten und einer stärkeren Arzneimittelexposition haben Frauen im Durchschnitt eine höhere Lebenserwartung als Männer. In dem äußerst komplexen Gefüge von Lebensbedingungen, Gesundheitszustand und Gesundheitsverhalten ergibt sich die Frage, ob und in welchem Maße die Arzneimittelexposition nicht nur auf den Krankheitsverlauf, sondern auch auf die Lebensdauer bzw. Lebensqualität Einfluß hat. Trägt die höhere Anwendungsfrequenz von Arzneimitteln zu der für Frauen längeren durchschnittlichen Lebensdauer bei oder wären die geschlechtsspezifischen Unterschiede für die Frauen noch günstiger, wenn die Arzneimittelexposition der weiblichen Bevölkerung nur genau so hoch oder gar geringer als die der Männer wäre? Diese Fragen sind jedoch nur mit einem Studienansatz zu beantworten, bei dem der zeitliche Verlauf von Exposition und Wirkungen angemessen berücksichtigt werden kann.

\section{Literatur:}

[1] Schwabe, U. (Hrsg.): Arzneiverordnungsreport '97 (Verfügungsbeklagte Ausgabe). 
Stuttgart, Jena, Lübeck, Ulm: Gustav Fischer Verlag 1997.

[2] IMS Research Group, IMS America, Ambler, Pennsylvania USA 1979.

[3] Tätigkeitsbericht 1991 des Bundesgesundheitsamtes, München: MMV Medizin Verlag 1992.

[4] Tätigkeitsbericht 1992 des Bundesgesundheitsamtes, München: MMV Medizin Verlag 1993.

[5] Tätigkeitsbericht 1993 des Bundesgesundheitsamtes, München: MMV Medizin Verlag 1994.

[6] Tätigkeitsbericht 1994 des Robert Koch-Instituts, München: MMV Medizin Verlag 1995.

[7] Tätigkeitsbericht 1995 des Robert Koch-Instituts, München: MMV Medizin Verlag 1996.

[8] Melchert, H.-U.: Einnahme von Psychopharmaka. In: Bellach, B.-M. (Hrsg.): Die Gesundheit der Deutschen. Robert Koch-Institut, Berlin 1995.

[9] Melchert, H.-U., und Linopf, H.: Daten der Nationalen Untersuchungs-Surveys zum Verbrauch oraler Kontrazeptiva (OC) in
West- und Ostdeutschland. In: Bellach, B.-M (Hrsg.): Die Gesundheit der Deutschen. Robert Koch-Institut 1996.

[10] Melchert, H.-U., Görsch, B., und Hoffmeister, H.: Nichtstationäre Arzneimittelanwendung und subjektive Arzneimittelverträglichkeit in der bundesdeurschen Wohnbevölke rung der 25-69jährigen - Ergebnisse der Erhebung des ersten Nationalen UntersuchungsSurveys 1984-1986. RKI-Schrift 1/95, München: MMV Medizin Verlag 1995.

[11] Krause-Girth, C.: Schein-Lösungen - Die Verschreibungspraxis von Psychopharmaka Bonn: Psychiatrie-Verlag 1989.

[12] Deutsche Hauptstelle gegen die Suchtgefahren (Hrsg.): Medikamentenabhängigkeit Freiburg im Breisgau: Lambertus-Verlag 1992.

[13] Knopf, H., Braemer-Hauth, M., Melchert, H.-U., und Thefeld, W.: Ergebnisse der Nationalen Untersuchungs-Surveys zum Laxanzienverbrauch, Bundesgesundhbl. 38 12 (1995) 459-467.

[14] Melchert, H.-U, and Pabel, E.: The Tocopherol Pattern in Human Serum Is Markedly Influenced by Intake of Vitamin E-
Drugs - Results of the German National Health Surveys - Journal of the American Oil Chemists Society 75, 2 (1998) 213-216.

[15] Melchert, H.-U., Knopf, H., and Gerding, B.: Coffeine concentration in Human serum in Dependence o Medication, Coffee consumption and Socieodemographic Variables - Results of the German National Health surveys - Pharmacoepidemiology and Drug Safety 7 Suppl. 2 (1998) 121.

[16] Melchert, H.-IJ., Knopf, H., and Pabel, E.: Use of Acetylsalicylic Acid-containing Drugs in Germany: Serum Levels of Salicylic Acid in Participants of National and Regional Surveys. Pharmacoepidemiology and Drug Safety 7 Suppl.2 (1998) 202-203.

[17] Kleinebrecht, J., Fränz, J, und Windorfer, A. Arzneimittel in der Schwangerschaft und Stillzeit. Stuttgart: Wissenschaftliche Verlagsgesellschaft 1995.

Anschrifr der Verfasser:

Dr. Hildtraud Knopf, Dr. Hans-Ulrich Melchert, Robert Koch-Institut, Postfach 6502 80, 13302 Berlin

\section{Schützt Stillen vor atopischem Ekzem?}

Grulee und Sandford [1] veröffentlichten 1936 eine Beobachtung an über 20000 Säuglingen, die bei Ernährung mit abgekochter Kuhmilch etwa sieben mal so häufig ein Ekzem in den ersten neun Lebensmonaten entwickelten wie ausschließlich gestillte Kinder. Allerdings hatten die Autoren damals alle Hauteffloreszenzen, außer dem Kopfgneis, als Ekzem bezeichnet. Stillen wird seither als wirksame Präventionsmaßnahme gegen Ekzem (Neurodermitis, Milchschorf) empfohlen. Dies ist durchaus berechtigt angesichts der beeindruckenden immunologischen Eigenschaften von Muttermilch [2]. Die klinischen und epidemiologischen Beobachtungen, die nach der Pionierarbeit von Grulee und Sanford [1] publiziert wurden, waren allerdings nicht so eindeutig, wie die Theorie es erwarten ließ. Kramer

Tabelle 1: Prozentanteil der Mütter, die ihr Kind sechs Monate oder länger gestillt haben in Anhängigkeit von der Sozialschicht nach Winkler et al. [6]. Nach Daten der multizentrischen Allergiestudie MAS-90 [4]

\begin{tabular}{cccc}
\hline $\begin{array}{c}\text { Untere } \\
\text { Schicht }\end{array}$ & $\begin{array}{c}\text { Mittlere } \\
\text { Schicht }\end{array}$ & $\begin{array}{c}\text { Obere } \\
\text { Schicht }\end{array}$ & $\mathrm{p}$ \\
\hline 11 & 24 & 36 & 0.00000 \\
\hline
\end{tabular}

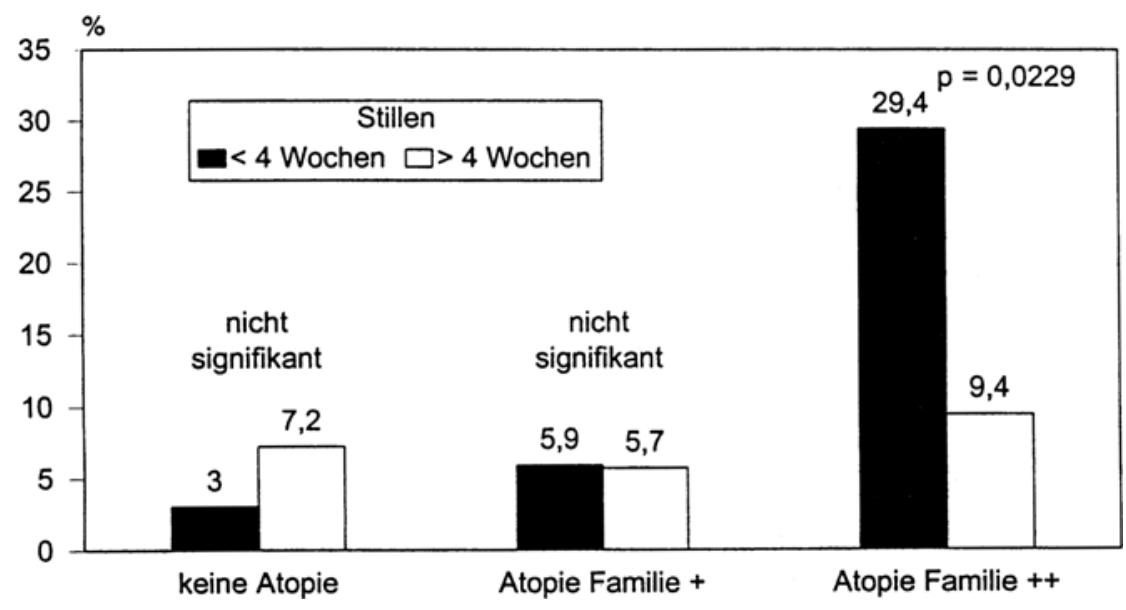

Abbildung 1: Prävalenz der Atopischen Dermatitis (A. D.) im Altersintervall ein bis drei Monate in Abhängigkeit von der Familienanamnese bei Säuglingen, die unter oder über vier Wochen gestillt worden waren. Atopie Familie + bzw. ++: Ein Atopiker bzw. mindestens zwei Atopiker in der engen Familie.

[3] fügte deshalb der Überschrift seiner Metaanalyse aller Publikationen zu diesem Thema hinzu: "Das goldenene Jubiläum einer Kontroverse«.

Warum konnten die einen Forschergruppen den präventiven Effekt des
Stillens gegen Neurodermitis bestätigen und die anderen nicht? Alle Beobachtungen hatten ihre Schwächen. Aber allen gemeinsam war, daß sie keine randomisierte Zuordnung der Probanden zum Stillen oder zu einer Flaschennahrung durchführen konn- 